\title{
ECUACIONES ALOMÉTRICAS PARA ÁRBOLES TROPICALES: APLICACIÓN AL INVENTARIO FORESTAL DE SINALOA, MÉXICO ${ }^{1}$
}

\author{
José Návar-Cháidez ${ }^{2}$,Felipa de Jesús Rodríguez-Flores ${ }^{3}$, Pedro Antonio Domínguez-Calleros ${ }^{4}$
}

\begin{abstract}
RESUMEN
Ecuaciones alométricas para árboles tropicales: aplicación al inventario forestal de Sinaloa, México. El objetivo del presente trabajo fue desarrollar una ecuación alométrica actualizada para la biomasa aérea total (M) de árboles medidos en campo y de sitios inventariados en los bosques secos de Sinaloa, México. Los resultados mostraron que: (a) la ecuación alométrica desarrollada incrementó la precisión en contraste con las ecuaciones convencionales de biomasa, (b) la alometría desarrollada ex situ proyecta valores de $\mathrm{M}$ que pueden desviarse por cerca del doble de las mediciones de M y (c) las evaluaciones al nivel del sitio también pueden desviarse por más del doble cuando se usan ecuaciones desarrolladas ex situ. Se probaron dos procedimientos para aumentar la precisión en la evaluación de M para árboles y sitios, cuando se ajustan ecuaciones desarrolladas ex situ: (i) con el teorema del límite central usar las ecuaciones alométricas disponibles para estimar un promedio y (ii) calibrar las ecuaciones por el ajuste de parámetros a nivel local, los cuales pueden ser calculados con el uso de programas estadísticos. Estas opciones pueden incrementar la precisión de las evaluaciones de M de árboles y sitios, y eventualmente de las selvas tropicales secas del norte de México.
\end{abstract}

Palabras clave: contraste de ecuaciones locales y fuera del sitio, Teorema del Límite Central, calibración de ecuaciones fuera del sitio.

\begin{abstract}
Tree biomass equations for tropical dry forests and their application to the Mexico's Sinaloan forest inventory. The research objective was to develop an updated allometric equation of the total aerial biomass (M) of trees measured on the field and inventory sites of Mexico's Sinaloan tropical dry forests. Results showed that: (a) the improved on site allometric equation increases precision in contrast to the conventional biomass equation previously reported, (b) deviations close to one order of magnitude occur between on site measurements of $\mathrm{M}$ and projections estimated with ex situ allometric equations, and (c) site evaluations may also deviate more than double when using ex situ allometric equations. Two procedures to increase tree and $\mathrm{M}$ precision when adjusting ex situ allometric equations were tested: (i) to use all available tree allometric functions to estimate a mean based on the central limit theorem or (ii) to calibrate ex situ equations by adjusting with local parameters through the use of statistical programs. These options would eventually increase tree and site estimations of total aerial biomass (M) in regional evaluations and eventually on dry forests of Northern Mexico.
\end{abstract}

Keywords: contrast analysis of on and off-site equations, Theorem of the Central Limit, local calibration of ex situ allometric equations.

\footnotetext{
Recibido: 13 de marzo, 2013. Aceptado: 28 de octubre, 2013. Este trabajo forma parte de las investigaciones realizadas en el marco de los proyectos CABSA-CONAFOR, 2003-2004.

2 Manejo de Recursos Naturales. Centro Interdisciplinario para el Desarrollo Integral Regional - Instituto Politécnico Nacional (CIIDIRIPN), Unidad Durango. Sigma No 119. Fraccionamiento 20 de Noviembre II. Durango, Dgo., México. 34220. jnavar@ipn.mx (autor para correspondencia).

3 Tecnología Ambiental. Universidad Politécnica de Durango.Carr. Mexico.Km 9.5. Dolores Hidalgo, Durango, México. jesu_rgz@ @otmail.com

4 Silvicultrura y Manejo Forestal. Facultad de Ciencias Forestales, Universidad Juarez del Estado de Durango (UJED). Rio Papaloapan S/n. Durango,Dgo., México.pdomingc@hotmail.com
} 


\section{INTRODUCCIÓN}

El desarrollo de ecuaciones alométricas es la metodología estándar para la estimación de la biomasa arbórea (Brown et al. 1989, Brown 1997, Chavé et al. 2003, 2005, 2006, Návar 2009a, 2010). Los manejadores y los investigadores de los recursos forestales requieren estimaciones fiables de la biomasa aérea total (M), para evaluar la abundancia de combustibles en un bosque, los efectos de los tratamientos aplicados a la cobertura forestal y la productividad del sitio en forma de madera y forraje, entre otros (Kie y White 1985, Harvey et al. 2011). La cantidad de biomasa aérea es importante en la evaluación de otros depósitos de biomasa ya que se correlaciona con la ubicada bajo el suelo, raíces, hojarasca y necro-masa (Cairns et al. 1997, Mokany et al. 2006). Estimaciones de la biomasa aérea son fundamentales para la estimación de la cantidad de bio-energía como alternativa parcial a los combustibles fósiles en la producción limpia y sostenible de la energía (McKendry 2002). El interés también se centra en las cuestiones ambientales mundiales porque los ecosistemas forestales contribuyen al ciclo global del carbono, y ayuda a mitigar los efectos del cambio climático (Canadell y Rapauch 2008). La evaluación de la biomasa aérea también contribuye a la comprensión de las teorías de asignación de recursos entre los órganos del árbol (West et al. 1999).

La ecuación más común reportada en la literatura científica es la convencional logarítmica en la que $\mathrm{M}$ se calcula como una función lineal logarítmica del diámetro normal (D) con los coeficientes de escala y pendiente (Ter-Mikaelian y Korzukhin 1997, Jenkins et al. 2003, Zianis y Mencuccini 2004, Návar 2009b). Esta es una ecuación empírica por naturaleza ya que los coeficientes escalares no son conocidos y se estiman generalmente por procedimientos de mínimos cuadrados en regresión lineal. Las ecuaciones actualizadas empíricas contienen el valor de la gravedad específica de la madera ( $\rho$ w) y la altura del dosel $(\mathrm{H})$ además del diámetro normal como variables independientes exógenas (Chavé et al. 2005, Návar 2009a). Un procedimiento totalmente teórico para estimar la biomasa se obtuvo utilizando la teoría de los fractales (West et al. 1999), en lo sucesivo denominado el modelo WBE ((West Biomass Equation), donde el principal supuesto es que $\mathrm{D}$ está relacionado con $\mathrm{M}$ por $\mathrm{M} \propto \mathrm{D} 8 / 3$, señalando que el exponente de escala $B_{\mathrm{wBE}}$ es igual a 8/3 =2,67 (West et al. 1999a, b, Enquist et al.
1998). Informes preliminares destacan que la técnica WBE tiene que ser mejorada antes de que pueda ser recomendado como un método de evaluación no destructiva de $\mathrm{M}$ porque se ha demostrado que el exponente de escala B es menor que 2,67 (Zianis y Mencuccini de 2004, Pilli et al. 2006, Návar 2009a).

Los modelos semi-empíricos son cada vez más comunes en la literatura científica (Návar 2010). Návar (2010) propuso y probó un procedimiento semi-empírico no destructivo flexible que utiliza el análisis de la forma dimensional. La metodología se ha probado para varias comunidades forestales del norte de México y resultó en la precisión dada por las ecuaciones de biomasa convencionales. Sin embargo, una mejor evaluación de $\mathrm{M}$ se proporciona por el modelo no destructivo que por otros modelos, el método es complicado, ya que requiere varias relaciones alométricas locales para deducir los coeficientes escalares apropiados. Návar (2010) propuso que el coeficiente escalar B es igual a 2,38 y la intersección a, es una función de $\rho w$, con un coeficiente de proporcionalidad C. Este último modelo fue probado con especies de árboles templados de América del Norte y proporciona un buen ajuste para los grupos de especies arbóreas propuestas por Jenkins et al. (2003).

Varios aspectos siguen siendo poco conocidos en la alometría de biomasa de los bosques tropicales. El objetivo del presente trabajo fue desarrollar una ecuación alométrica actualizada para la biomasa aérea total (M) de árboles medidos en campo y de sitios inventariados en los bosques secos de Sinaloa, México.

\section{MATERIALES Y MÉTODOS}

En este apartado se describe la teoría alométrica sobre ecuaciones de biomasa disponibles en la literatura científica universal. Esto se hace con el fin de presentar el marco de referencia en el cual se desarrollan y prueban las ecuaciones de biomasa a fuentes de datos de biomasa. Para entender mejor el marco se describen los modelos teóricos, semi-empíricos y empíricos clasificados por la forma en estimar sus parámetros por Návar (2010).

\section{Teoría alométrica}

Las ecuaciones de biomasa se pueden clasificar de acuerdo con la técnica empleada en la estimación de 
parámetros en modelos empíricos, semi-empíricos y teóricos (Návar 2010). Las ecuaciones empíricas ajustan los parámetros por mínimos cuadrados en regresión. Los modelos teóricos físicamente parameterizan las ecuaciones que expresan descripciones físicas de la biomasa. Los modelos semi-empíricos son técnicas no destructivas que requieren de parámetros físicos y estadísticos (Návar 2010).

El modelo teórico de West et al. (1999a). El modelo teórico no destructivo [1] fue propuesto por West et al. (1999a) y se desarrolló utilizando la teoría de los fractales, WBE. El marco WBE describe M del árbol con la siguiente ecuación:

$$
M=\mathrm{C} \rho D^{8 / 3}
$$

Donde: $\mathrm{C}=$ una constante de proporcionalidad, y $\rho=$ la gravedad específica de la biomasa en pie. El exponente escalar, B, se fija a 8,3 $=2,67$ y la gravedad específica se denomina como la gravedad específica total del árbol (un promedio ponderado de madera, corteza, ramas y hojas).

El modelo alométrico [2] describe físicamente $\mathrm{M}$ y fue propuesto preliminarmente por Návar (2010), como:

$$
M=\mathrm{CV} \rho
$$

Donde: $\mathrm{V}=$ volumen total árbol en pie $\left(\mathrm{m}^{3}\right) ; \rho$ = la densidad de la biomasa de los árboles en pie $\left(\mathrm{g} / \mathrm{cm}^{3} \mathrm{o} \mathrm{Mg} / \mathrm{m}^{3}\right)$, y $\mathrm{C}=$ factor de forma para objetos no-euclidianas.

El volumen del fuste convencionalmente se reporta en los inventarios forestales, aunque en ocasiones el volumen de las ramas no se incluye y tiene que calcularse utilizando otros métodos independientes. Por otro lado, los valores de gravedad específica para árboles enteros son poco conocidos y la mejor suposición es asumir que la gravedad específica de la madera, $\rho$ w, medido convencionalmente en estudios de tecnología de madera es igual a $\rho$. La forma de los fustes no sigue la geometría Euclidiana convencional y, por lo tanto, el factor de forma $\mathrm{C}$ trata de describir esta variabilidad.

Ecuaciones empíricas. Návar (2010) reportó el modelo empírico más común para estimar la biomasa aérea es la ecuación logarítmica [3]:

$$
\operatorname{Ln}(M)=\operatorname{Ln}(a) \pm B \operatorname{Ln}(\mathrm{D}) \pm \mathrm{e}_{\mathrm{i}}=a \mathrm{D}^{B}+\mathrm{e}_{\mathrm{i}}
$$

Donde: $a$ y $B$ son la intersección y el exponente (B) de la ecuación [3]; ambos parámetros se calculan generalmente por mínimos cuadrados en regresión lineal logarítmica; $\mathrm{e}_{\mathrm{i}}=$ el error. La intercepción del modelo [3]; $a=\mathrm{C} \rho$ del modelo [1] y $B$ del modelo $\mathrm{WBE}, \mathrm{B}_{\mathrm{wBE}}=2,67$. Note que $\mathrm{B}_{\mathrm{wBE}} \neq \mathrm{B}$.

Otras ecuaciones empíricas nuevas y modernas se expanden a partir de la ecuación [2] y contienen la gravedad específica de la madera y la altura del dosel, además del diámetro normal. Este tipo de ecuaciones fue propuesto por Chavé et al. (2005) para bosques tropicales, y por Návar (2009a) para los bosques templados del norte de México. Un ejemplo matemático de esta clase de modelos se presenta en el modelo [4]:

$$
M=\left\lfloor(p w \cdot a) D^{b} H^{h}\right\rfloor=\left\lfloor(p w \cdot a) \cdot\left(D^{2} H\right)^{h}\right\rfloor
$$

\section{Modelos semi-empíricos}

El modelo del exponente constante. Návar (2010) propuso que los coeficientes escalares promedio cercanos a la media de la población se encuentra en los estudios empíricos que compilan las ecuaciones de biomasa. Cuando se considera el diámetro normal, un valor promedio $\mathrm{B}=2,38$ ha sido reportado en estudios de meta-análisis de ecuaciones de biomasa (Návar 2010). El valor de la intersección a, es una función de la gravedad específica de la madera (Návar 2010). Con este supuesto, y una función estadística, el modelo reducido propuesto [5] es:

$$
M=\left[C p_{w}\right] D^{2,38}
$$

El valor de C estimado es de 0,2457 para especies de árboles de clima templado de América del Norte, y de 0,2593 para las especies templadas mundiales reportadas por Zianis y Mencuccini (2004), de acuerdo con cálculos desarrollados por Návar (2010).

El modelo de la forma dimensional. Návar (2010) desarrolló el modelo semi-empírico no destructivo [6] utilizando las relaciones dimensionales de forma del fuste: 


$$
M=[a=f(B)] D^{B=\left(d+h B^{*}\right)}
$$

Donde: $a, B=$ la intercepción y el exponente escalar, respectivamente, $d, h=$ los coeficientes de la ecuación de Schumacher y Hall (1933), B* = el coeficiente de la relación logarítmica de la altura con el diámetro, $\mathrm{H}=\mathrm{f}(\mathrm{D})$.

El modelo [6] tiene la ventaja de la buena relación existente entre los coeficientes escalares como ha sido reportado en la literatura científica (Zianis y Mencuccini 2004).

\section{Los datos de campo}

El bosque seco sinaloense cubre una superficie de $77500 \mathrm{~km}^{2}$ en los estados mexicanos de Sonora, Sinaloa y Nayarit. Se distribuye desde la base de la Sierra Madre Occidental hacia el oeste hasta el Océano Pacífico. En esta zona climática se distribuye el bosque tropical seco con las especies comúnmente encontradas: Lysiloma divaricata, Haematoxylon brasiletto, Bursera penicillatum, Erythrina guatemalensis, Guazuma ulmifolia, Ipomea arborescens, Ceiba acuminata, Pithecellobium manganese y Rubus palmeri, entre otras. Los detalles de la muestra de datos para los 39 árboles cortados se pueden encontrar en NávarCháidez (2009). Los valores de gravedad específica de madera de estas especies fueron recolectadas principalmente de la lista reportada por Chavé et al. (2005). Con estos datos se ajustó la ecuación moderna que incluye el diámetro normal, la altura total y la gravedad específica de la madera. Los datos de inventario de 390 parcelas con dimensiones $(20 \times 50 \mathrm{~m})$ distribuidas en los ejidos Vado Hondo y San José de Tiniaquis, Sinaloa, México estuvieron disponibles para el ajuste de las ecuaciones desarrolladas in situ y ex situ.

\section{Ecuaciones alométricas probadas}

Un total de once ecuaciones, dos in situ y nueve ex situ (siete empíricas, dos semi-empíricas y dos teóricas) se ajustaron a la biomasa aérea total medida para los 39 árboles provenientes de bosques tropicales secos (Cuadro 1).

Las ecuaciones de Návar (2010) y de West et al. $(1999 a, b)$ son de la misma naturaleza, con las excepciones señaladas anteriormente. El método no destructivo de Návar (2010) utiliza el $\mathrm{H}=\mathrm{f}(\mathrm{D})$ y $\mathrm{V}=\mathrm{f}(\mathrm{H}, \mathrm{D})$ o así como una ecuación empírica para estimar el coeficiente B. El modelo de West et al. (1999a, b) supone

Cuadro 1. Ecuaciones alométricas utilizadas para probar la biomasa aérea en pie de 39 árboles muestreados destructivamente en un bosque tropical seco de Sinaloa, México. 2013.

\begin{tabular}{|c|c|c|c|}
\hline Investigador & Bosque tropical & Modelo & Ecuación \\
\hline Brown (1997) & Seco & $\mathrm{E}-\mathrm{C}$ & $\mathrm{M}=34,47-8,0671 \mathrm{D}+0,6589 \mathrm{D}^{2}$ \\
\hline Brown (1997) & Seco & $\mathrm{E}-\mathrm{C}$ & $M=\exp (-1,996+2,32 \operatorname{Ln}(D))$ \\
\hline Martínez-Yrizar et al. (1992) & Seco & $\mathrm{E}-\mathrm{C}$ & $\mathrm{M}=10^{(-0,5352+\log 10(\mathrm{BA}))}$ \\
\hline Ecuación moderna & Seco & E-U & $\mathrm{M}=0,08479\left(\rho_{\mathrm{w}}^{0,55255} \mathrm{D}^{2,2435} \mathrm{H}^{0,4773}\right)$ \\
\hline Ecuación convencional & Seco & E-C & $\mathrm{M}=\mathrm{e}\left(-2,409+0,952^{*} \operatorname{Ln}\left(\mathrm{D}^{2} \mathrm{H}\right)\right)$ \\
\hline Chavé et al. (2005) & Seco & E-U & $\mathrm{M}=0,112 *\left(\rho_{\mathrm{w}} \mathrm{D}^{2} \mathrm{H}\right)^{0,916}$ \\
\hline Chavé et al. (2005) & Seco & E-U & $\mathrm{M}=\rho_{\mathrm{w}}{ }^{*} \mathrm{e}\left(-0,67+1,78 \operatorname{Ln}(\mathrm{D})+0,207(\ln (\mathrm{D}))^{2}-0,028(\operatorname{Ln}(\mathrm{D}))^{3}\right)$ \\
\hline Návar (2010) & Todos & S-E-C & $\left.\mathrm{M}=\left(38,36^{*} \mathrm{~B}^{-6,9045}\right) \mathrm{D}^{(\mathrm{B}=\mathrm{d}+\mathrm{hB}} *\right)$ \\
\hline Návar (2010) & Todos & S-E-U & $M=\left(0,2457 \rho_{w}\right) D^{2,38}$ \\
\hline West et al. $(1999 \mathrm{a}, \mathrm{b})$ & Todos & $\mathrm{T}$ & $\mathrm{M}=0,2457 \rho_{\mathrm{w}}\left(\mathrm{D}^{2,67}\right)$ \\
\hline Návar (2010) & Todos & $\mathrm{T}$ & $\mathrm{M}=\mathrm{CV}^{*} \rho_{\mathrm{w}}$ \\
\hline
\end{tabular}

Donde: $\mathrm{D}=$ diámetro normal, $\mathrm{H}=$ altura total, $\mathrm{BA}=$ área basal, $\rho_{\mathrm{w}}=$ gravedad específica de la madera, $\mathrm{E}-\mathrm{C}=$ empírica convencional, $\mathrm{E}-\mathrm{U}=$ empírico-actualizado, S-E-C= semi-empírico-convencionales, S-E-U= semi-empírico-actualizado no destructivo, T= modelos teóricos, Todos $=$ todos los bosques, ya que son modelos teóricos y semi-empíricos por naturaleza. 
que $\rho=\rho$ w y $\mathrm{C}=$ una constante de proporcionalidad. Los valores del coeficiente de $\mathrm{C}$ están disponibles en la literatura científica (Návar 2010); sin embargo, estos no se han reportado para los bosques tropicales. Por lo tanto, el valor del coeficiente C se estimó en tres formas diferentes: (a) buscando iterativamente el valor C; (b) calculando un valor medio de la ecuación: $\mathrm{C}=$ $\mathrm{M} /\left(\rho \mathrm{w} \mathrm{D}^{2,67}\right)$, y (c) por el cálculo estadísticamente en regresión no lineal. La ecuación de la física también supone que $\rho=\rho \mathrm{w}$. La ecuación convencional fue reportada previamente por Návar-Cháidez (2009).

Las ecuaciones de Brown (1997), Martínez-Yrizar (1992) y la reportada previamente en Návar-Cháidez (2009) son de naturaleza convencional; la ecuación moderna, las de Chavé et al. (2005), la de Návar (2010), la de la física de masas, y la de West et al. (1999a, b), son modelos actualizados ya que contienen otras variables exógenas, además del diámetro normal. Todas estas ecuaciones se ajustaron a los datos del inventario forestal para los 390 sitios de 0,10 ha de superficie para entender las fuentes de variabilidad inherentes a $\mathrm{M}$.

En la búsqueda de una ecuación alométrica que resulte con un error estándar similar a aquel de la ecuación local, se promediaron los valores de las ecuaciones ex situ y semi-empíricas y se volvió a calcular M y el error estándar. Esta hipótesis se basa en el Teorema del Límite Central en donde los promedios se aproximan más que los valores individuales al promedio universal y este principio fue probado anteriormente con éxito por Návar (2010) para especies templadas de México.

Dos fuentes de error o nivel de precisión: a) la desviación estándar y b) la desviación estándar como porcentaje o coeficiente de variación, describen la heterogeneidad inherente a las estimaciones de biomasa aérea por todas las ecuaciones. La ecuación [7] calcula la desviación estándar, Sxe, a través de las diferentes ecuaciones alométricas árbol:

$$
S x e=\sqrt{\frac{\sum_{i=1}^{n}(M i-\bar{M})^{2}}{n-p-1}}
$$

Dónde: $M i=$ biomasa aérea promedio estimado por la ecuación alométrica i (kg/árbol), $\bar{M}=$ promedio medido de $\mathrm{M}$ ( $\mathrm{kg} /$ árbol), y $n=$ número de observaciones, $p=$ número de parámetros estimados para cada ecuación alométrica.
La desviación estándar en porcentaje, $S x(\%)$ se calculó como una función de la media de la biomasa aérea de todas las ecuaciones alométricas utilizados por la ecuación [8]:

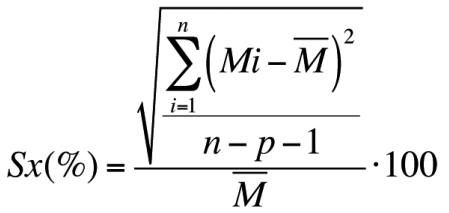

Donde: $\bar{M}=$ promedio de $\mathrm{M}(\mathrm{kg})$.

\section{RESULTADOS Y DISCUSIÓN}

La ecuación moderna con la mejor bondad de ajuste fue: $\mathrm{M}=0,08479\left(\rho \mathrm{w} 0,55255 \mathrm{D}^{2,2435} \mathrm{H}^{0,4773}\right)$, donde: $\rho \mathrm{w}=$ gravedad específica de la madera; $\mathrm{D}=$ diámetro normal $(\mathrm{cm}), \mathrm{H}=$ altura total $(\mathrm{m})$. Los estadísticos de ajuste error estándar, Sx; el error estándar en porcentaje, Sx (\%), y el coeficiente de determinación, $\mathrm{r}^{2}$, para esta ecuación son: $34,7 \% ; 40,62 \%$, y 0,88 , respectivamente.

Las once ecuaciones presentan diferente grado de ajuste y seis de ellas predicen valores individuales de $\mathrm{M}$, mientras que las otras cinco ecuaciones reportan valores promedio de $\mathrm{M}$ en función del diámetro normal (Figura 1). Es decir, las ecuaciones alométricas con las variables exógenas diámetro normal, altura total, gravedad específica de la madera predicen valores cambiantes de $\mathrm{M}$ sin seguir una línea recta en función de D. Las ecuaciones convencionales que contienen solamente el diámetro normal proporcionan proyecciones de $\mathrm{M}$ solo en función de $\mathrm{D}$ siguiendo una ecuación de potencia (Figura 1).

Las fuentes de error como la desviación estándar el coeficiente de variación (cv) muestran que las ecuaciones alométricas desarrolladas in situ proporcionan la mejor bondad de ajuste $\left(\mathrm{r}^{2}=0,88\right.$ y 0,78 y los $\mathrm{cv}$ de $40,6 \%$ y $52,5 \%$ en contraste con las ecuaciones convencionales desarrolladas ex situ) (Cuadro 2).

Siete diferentes ecuaciones ex situ sesgaron las estimaciones de la biomasa aérea total para la especie Leucaena leucocephala de Hawaii (Youkhana e Idol 2011); donde estos autores probaron siete diferentes ecuaciones alométricas de biomasa ex situ y todas sesgaron M. Una posible fuente de variación se puede explicar por la falta de valores de gravedad específica de la madera medidos in situ, ya que estos valores varían 

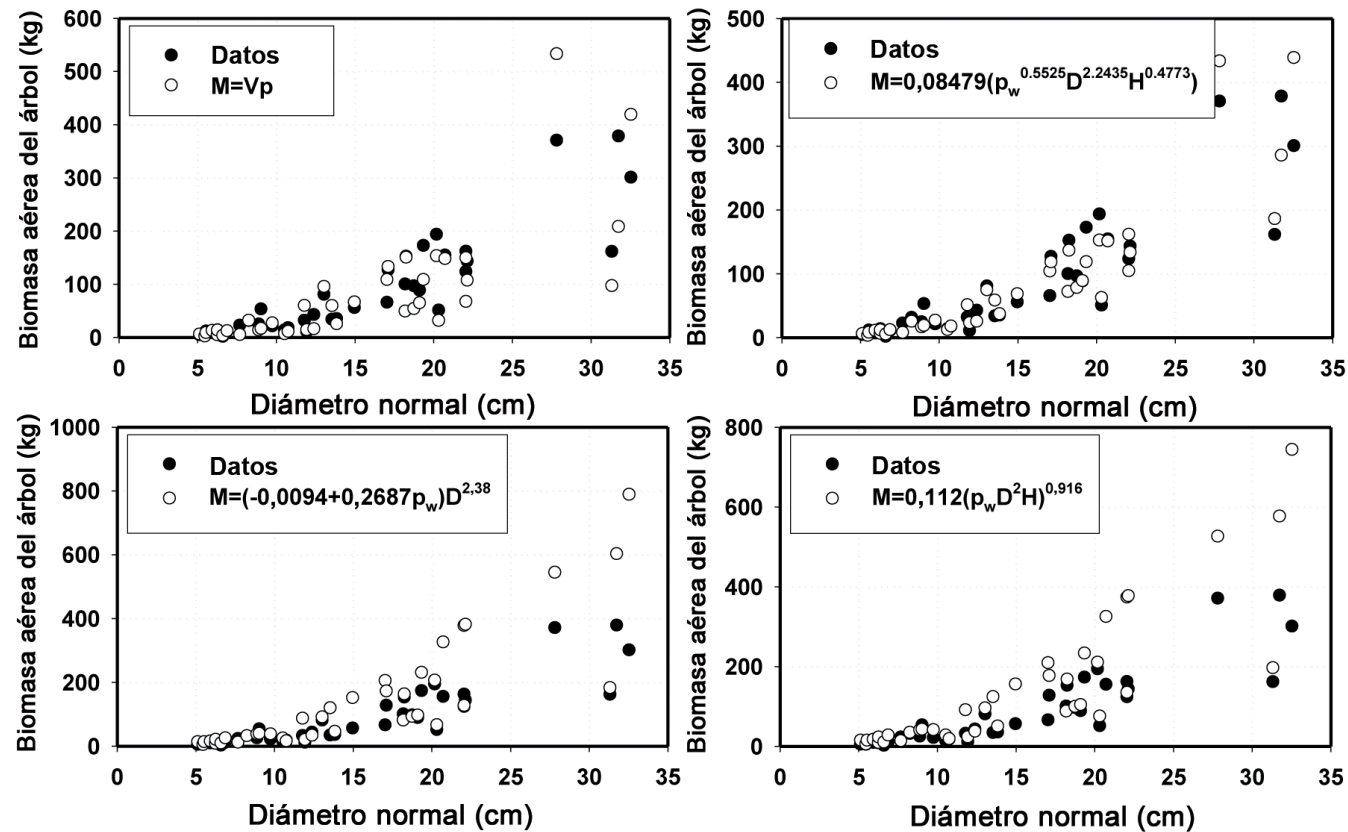

Diámetro normal $(\mathrm{cm})$
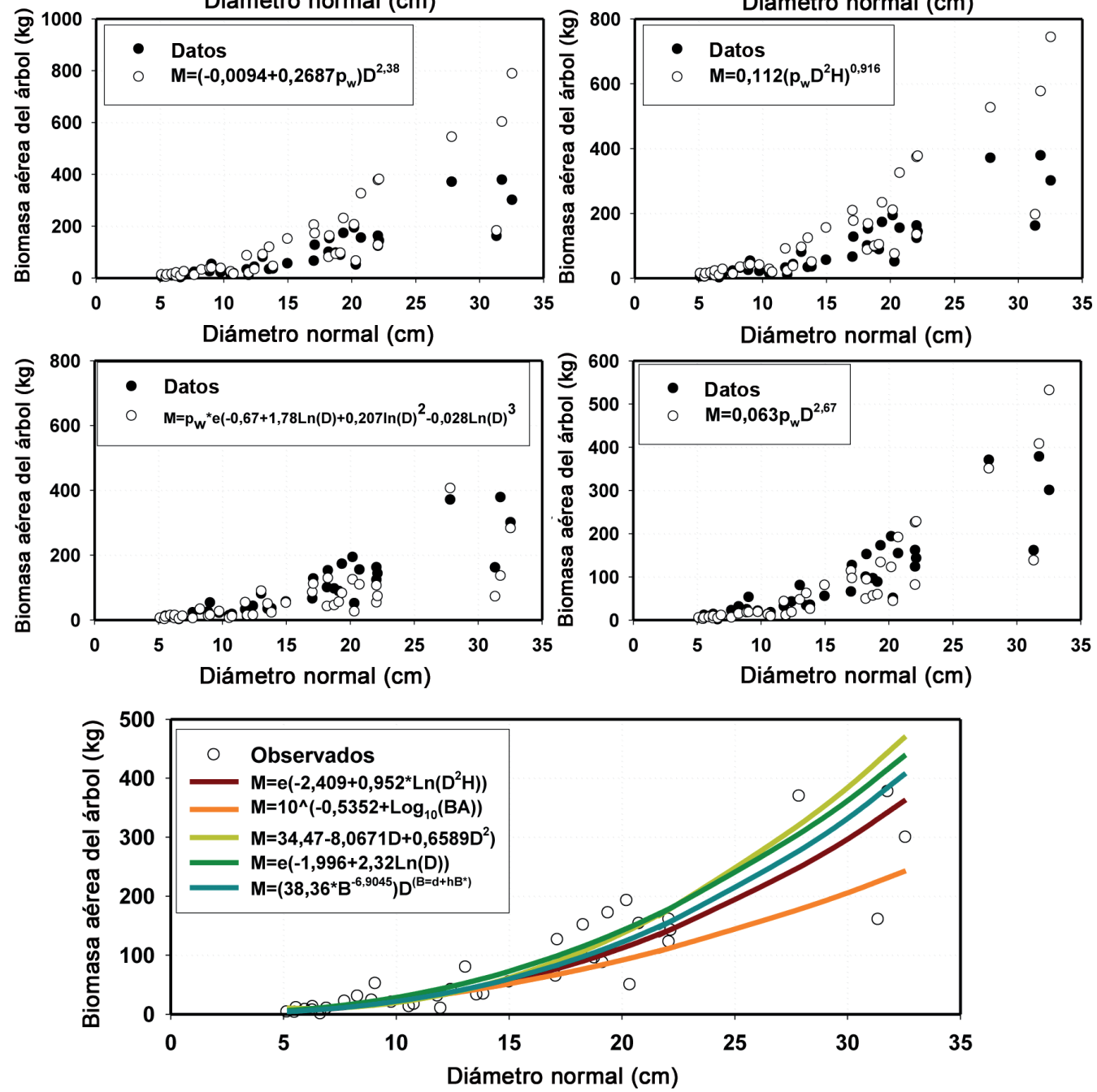

Figura 1. Valores de biomasa aérea de árboles medidos y estimados con once ecuaciones para bosques tropicales secos de Sinaloa, México. 2005, 2013. 
Cuadro 2. Estadísticos de bondad de ajuste de once ecuaciones ajustadas a los datos de biomasa aérea medidos en bosques tropicales secos de Sinaloa, México. 2013.

\begin{tabular}{lccccc}
\hline Investigador & Bosque tropical & Clase de modelos & $\mathbf{R}^{2}$ & \multicolumn{2}{c}{ Desviación estándar } \\
\cline { 5 - 6 } & & & & (kg/árbol) & $(\%)$ \\
\hline Brown (1997) & Seco & E-C & 0,61 & 60,00 & 70,14 \\
Brown (1997) & Seco & E-C & 0,68 & 54,60 & 63,83 \\
Martínez-Yrizar et al. (1992) & Seco & E-C & 0,70 & 52,53 & 61,41 \\
Moderna & Seco & E-U & 0,88 & 34,75 & 40,62 \\
Convencional & Seco & E-C & 0,78 & 44,93 & 52,52 \\
Chavé et al. (2005) & Seco & E-U & 0,26 & 110,80 & 129,53 \\
Chavé et al. (2005) & Seco & E-U & 0,69 & 54,99 & 64,28 \\
Návar (2010) & Todos & S-E-C & 0,74 & 50,21 & 58,70 \\
Návar (2010) & Todos & S-E-U & 0,41 & 114,37 & 133,70 \\
West et al. (1999a, b) & Todos & T & 0,73 & 50,16 & 58,64 \\
Física (Návar 2010) & Todos & T & 0,73 & 49,57 & 57,95 \\
Valores promedio para todas las ecuaciones fuera del sitio & 0,62 & 66,35 & 77,58 \\
\hline
\end{tabular}

E-C = ecuación convencional; E-U = ecuación universal; S-E-C = ecuación semi-empírica convencional; S-E-U = ecuación semiempírica universal; E-T = ecuación teórica; $\mathrm{T}$ = todos los bosques tropicales.

entre los árboles de la misma especie (Parolin 2002, Chavé et al. 2006, Silva-Arredondo y Návar 2009), además de que los valores medios fueron adquiridos de las listas de Chavé et al. (2006).

La ecuación generalista mundial de Chavé et al. (2005) fue inferior a las ecuaciones locales. Las otras presentaron estadísticas de ajuste menores a los que presenta la ecuación convencional desarrollada in situ. Estas son en orden descendente de bondad de ajuste: (a) la ecuación semi-empírica derivada de la forma dimensional propuesta por Návar (2010), (b) la ecuación de la física de masas (Návar 2010), (c) el modelo teórico WBE (West et al. 1999a, b). Todas estas ecuaciones reportaron coeficientes de determinación por encima de 0,73 y la desviación estándar de menos de $50,5 \mathrm{~kg} /$ árbol. Estas ecuaciones tienen un componente teórico y la primera es de naturaleza convencional.

Las ecuaciones alométricas restantes, los modelos convencionales (Brown 1997, Martínez-Yrizar et al. 1992) y la ecuación moderna (Chavé et al. 2005) producen desviaciones $>52,5 \mathrm{~kg} /$ árbol, y coeficientes de determinación $<0,70$. Dos ecuaciones resultaron en estimaciones de $\mathrm{M}$ estadísticamente diferentes; la ecuación de Chavé et al. (2005) con un $\mathrm{r}^{2}$ de 0,26 y una desviación estándar de 110 kg/árbol, y el modelo semiempírico no destructivo que supone una constante B propuesto por Návar (2010) con un $\mathrm{r}^{2}$ de 0,41 y una

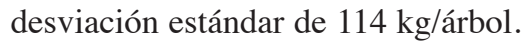

Por lo tanto, se debe tener cuidado al seleccionar una ecuación para estimar M, ya que las evaluaciones pueden subestimar (Chavé et al. 2005.) o sobreestimar M por cerca de una orden de magnitud (Chavé et al. 2005, Návar 2010). Es decir, mientras que el promedio de $\mathrm{M}$ es $85,5 \mathrm{~kg} /$ árbol, las ecuaciones de Chavé et al. (2005) y Návar (2010) proyectan en promedio 60,7 y 139,5 y $140,7 \mathrm{~kg} /$ árbol, respectivamente.

La biomasa aérea promedio al nivel del sitio proyectada por los once modelos se representan en la Figura 2. Los límites de confianza muestran que ambas ecuaciones de Brown (1997) predicen evaluaciones compatibles de $\mathrm{M}$ al nivel del sitio con la ecuación moderna desarrollada en este trabajo. Otras ecuaciones alométricas desarrolladas ex situ sub (Martínez-Yrizar et al. 1992, Chavé et al. 2005) o sobre (Návar 2010) estiman $\mathrm{M}$ al nivel de la parcela por más de una orden de magnitud. Cuando se selecciona una ecuación desarrollada ex situ para evaluar M al nivel de la parcela, se debe tener en cuenta los potenciales errores y sesgos. 


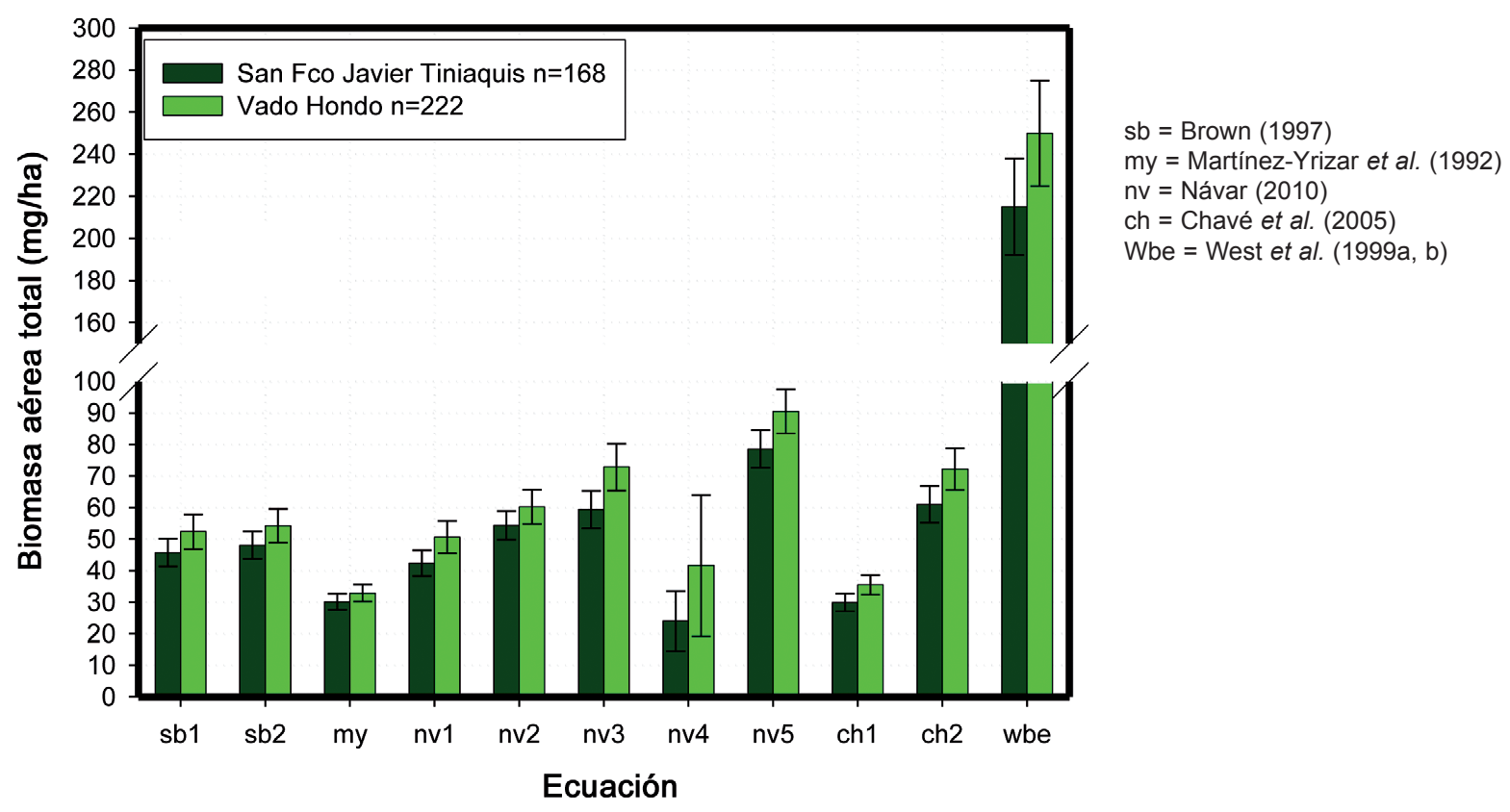

Figura 2. Valores de biomasa aérea derivadas de la aplicación de once ecuaciones al inventario forestal de los bosques tropicales secos de dos ejidos de Sinaloa, México. 2013.

La fuente más importante de error en la estimación de $\mathrm{M}$ a la escala del árbol, parcela o regional es la elección de un modelo alométrico (Chavé et al.2004, 2005, Houghton 2005a, Návar 2010). Los resultados muestran que las evaluaciones de $\mathrm{M}$ a la escala del árbol, pueden estar sesgadas por cerca de una orden de magnitud, para bosques tropicales secos de Sinaloa. Con antecedentes estadísticos, Návar (2010) planteó la hipótesis de que la estimación de M calculada con una combinación de al menos dos ecuaciones independientes podría mejorar las evaluaciones más allá de cualquier modelo alométrico individual desarrollado ex situ. El Cuadro 3 muestra los resultados de probar que esta hipótesis es correcta también para los estudios de casos descritos a continuación. Los datos para árboles agroforestales de Leucaena leucocephala

Cuadro 3. Prueba de la hipótesis de que los valores medios obtenidos a partir de combinaciones de ecuaciones proporcionan estadísticos de mejor ajuste a los datos de biomasa medidos en bosque tropical seco de Sinaloa, México que las ecuaciones individuales desarrolladas ex situ. 2013.

\begin{tabular}{|c|c|c|c|c|}
\hline \multirow{3}{*}{ Clase de ecuación alométrica } & \multirow{3}{*}{ Ecuaciones combinadas } & \multicolumn{3}{|c|}{ Coeficiente de variación } \\
\hline & & \multirow[t]{2}{*}{$\mathbf{r}^{2}$} & \multicolumn{2}{|c|}{ Desviación estándar } \\
\hline & & & (kg/árbol) & $(\%)$ \\
\hline Convencional & Brown (1997), Martínez et al. (1992) & 0,77 & 47,80 & 55,84 \\
\hline Convencional-moderna & Chavé et al. (2005) & 0,74 & 51,08 & 59,71 \\
\hline Semi-empírica, no-destructiva & Návar (2010) & 0,75 & 49,78 & 58,20 \\
\hline Convencional, semi-empírica y teórica & Todas las ecuaciones fuera del sitio & 0,84 & 40,47 & 47,32 \\
\hline \multicolumn{2}{|c|}{ Valores promedio } & 0,78 & 47,28 & 55,26 \\
\hline
\end{tabular}


de Hawaii mejoran el ajuste al agrupar juntos las siete ecuaciones desarrolladas fuera de sitio, aunque sesgan todavía ligeramente $\mathrm{M}$ (Youkhana e Idol 2011).

Todos los estadísticos de bondad de ajuste mejoran cuando se combinaron más de dos ecuaciones. Por ejemplo, utilizando los valores medios presentados en los Cuadros 2 y 3 , el coeficiente de determinación aumenta en promedio $25 \%$ al juntarse más de dos ecuaciones y alcanza un valor cercano a la ecuación empírica-moderna desarrollada in situ para este estudio cuando se combinan todas las ecuaciones alométricas ex situ. La desviación estándar se reduce en $40 \%$ cuando se mezclan al menos dos ecuaciones alométricas. Por lo tanto, en ausencia de alometría local, como es el caso de muchos bosques secos tropicales, la combinación de ecuaciones alométricas desarrolladas ex situ mejoraría las evaluaciones de M, de acuerdo con las hipótesis del teorema del límite central. Es recomendable tener presente las ecuaciones mundiales, así como los modelos alométricos semi-empíricos y teóricos disponibles. Estos modelos deben ser calibrados localmente, así como realizar un promedio de estos para producir mejores estimadores de $\mathrm{M}$.

Las ecuaciones individuales pueden ser localmente calibradas para utilizarlas en la evaluación de M para bosques tropicales secos sinaloenses. Por ejemplo, la ecuación semi-empírica no destructiva reducida que supone una constante B mejora su $\mathrm{r}^{2}$ de 0,41 a 0,83 y reduce su desviación estándar de 114,27 a 40,22 kg/ árbol si el coeficiente $\mathrm{C}$ de 0,2457 es sustituido por el de 0,135 . Ketterings et al. (2001) reportaron un valor de $\mathrm{C}$ de 0,11 para los árboles tropicales del sudeste de Asia. Sin embargo, en la evaluación de $\mathrm{M}$ al nivel del sitio, el nuevo valor de $\mathrm{C}$ que coincide con las estimaciones derivadas de la ecuación actualizada debe tener un valor de $\mathrm{C}$ de 0,175 . El valor de $\mathrm{C}=$ 0,175 es consistente para ambas fuentes de datos del inventario forestal (San Fco Javier Tiniaquis $\mathrm{n}=168 \mathrm{y}$ Vado Hondo $n=222$ ). El valor de $C$ se calculó con un programa estadístico de regresión no lineal. Mientras que otras estimaciones del coeficiente $\mathrm{C}$, aplicando otras estrategias (por ejemplo, $\mathrm{C}=\mathrm{M} /\left(\rho w \mathrm{D}^{2,38}\right)$ ) o por ensayo y error produce mejores estimaciones de $\mathrm{M}$, el cálculo estadístico resultó en las mejores aproximaciones de $\mathrm{M}$ y garantiza los estadísticos que se producen cuando se aplica la ecuación actualizada a este conjunto de datos. El modelo de Chavé et al. (2005) (por ejemplo, $\mathrm{M}=0,112^{*}\left(\rho w \mathrm{D}^{2} \mathrm{H}\right)^{0,916}$ ) recupera el valor de $\mathrm{r}^{2} 0,69$ a 0,82 si los parámetros del modelo se sustituyen (por ejemplo, $\left.\mathrm{M}=0,1847^{*}\left(\rho \mathrm{wD}^{2} \mathrm{H}\right)^{0,8881}\right)$. Las evaluaciones de $\mathrm{M}$ al nivel de la parcela con este nuevo conjunto de parámetros se ajustaron a aquellos estimados por la ecuación alométrica moderna actualizada. El segundo modelo de Chavé et al. (2005) (por ejemplo, $\quad \mathrm{M}=\rho \mathrm{w}^{*} \mathrm{e}\left(-0,67+1,78 \mathrm{Ln}(\mathrm{D})+0,207 \mathrm{Ln}(\mathrm{D})^{2}-\right.$ $\left.0,028 \mathrm{Ln}(\mathrm{D})^{3}\right)$ sólo se puede mejorar mediante el ajuste de la función exponencial simple (por ejemplo, $\mathrm{M}=\rho \mathrm{w}^{*}$ $\mathrm{e}(-1,92899+2,4226 * \operatorname{Ln}(\mathrm{D}))$ con un valor de $\mathrm{r}^{2}$ de 0,83 en lugar del 0,29 calculado previamente. El uso de este nuevo conjunto de parámetros produce evaluaciones de M compatibles entre el modelo de Chavé et al. (2005) y el moderno actualizado desarrollado para este trabajo.

La ecuación moderna desarrollada localmente proporcionó la mejor bondad de ajuste en contraste con los modelos calibrados localmente o que cualquier combinación de las ecuaciones desarrolladas ex situ. Por lo tanto, es recomendable cosechar destructivamente árboles para desarrollar o adaptar alometría al nivel del árbol in situ. Para bosques tropicales secos, se recomienda cosechar árboles en el rango de los diámetros mayores de las especies dominantes que se encuentran en estos bosques, ya que la mayor variación se encuentra en el lado derecho de la ecuación alométrica; es decir, en los árboles con los diámetros normales mayores.

\section{AGRADECIMIENTOS}

Se agradece a los revisores anónimos para mejorar la legibilidad y contenido técnico de este manuscrito. El autor principal de este trabajo es becario de la Comisión de Operaciones y Fomento de Actividades Académicas (COFAA) del Instituto Politécnico Nacional (IPN).

\section{LITERATURA CITADA}

Brown, S. 1997. Estimating biomass and biomass change of tropical forests. Forest Resources Assessment Publication. Forestry Papers 134. Rome, Italy, FAO. $55 \mathrm{p}$.

Brown, S; Gillespie, AJ; Lugo, AE. 1989. Biomass estimation methods for tropical forests with applications to forest inventory data. Forest Science 35:881-902.

Cairns, MA; Brown, S; Helmer, EH; Baumgardner, GA. 1997. Root biomass allocation in the world's upland forests. Oecologia 111:1-11. 
Canadell, JG; Rapauch, MR. 2008. Managing forests for climate change mitigation. Science 320:1456-1457.

Chavé, J; Condit, R; Lao, S; Caspersen, JP; Foster, RB; Hubbell, SP. 2003. Spatial and temporal variation in biomass of a tropical forest: results from a large census plot in Panama. Journal of Ecology 91:240-252.

Chavé, J; Condit, R; Aguilar, S; Hernández, A; Lao, S; Pérez, R. 2004. Error propagation and scaling for tropical forest biomass estimates. Phil. Trans. R. Soc. Lond. B. DOI 10.1098/rstb.2003.1425.

Chavé, J; Andalo, C; Brown, S; Cairns, MA; Chambers, JQ; Eamus, D; Folster, H; Fromard, F; Higuchi, N; Kira, T; Lescure, JP; Nelson, BW; Ogawa, H; Puig, H; Riera, B; Yamakura, T. 2005. Tree allometry and improved estimation of carbon stocks and balance in tropical forests. Oecologia 145(1):87-99.

Chavé, J; Muller-Landau, HC; Baker, TR; Easdale, TA; Steege, H; Webb, CO. 2006. Regional and phylogenetic variation of wood density across 2456 neotropical tree species. Ecological Applications 16:2356-2367.

Enquist, BJ; Brown, JH; West, GB. 1998. Allometric scaling of plant energetics and population density. Nature 395:163-165.

Harvey, CA; Villanueva, C; Esquivel, H; Gómez, R; Ibrahim, M; López, M; Sinclair, FL. 2011. Conservation value of dispersed tree cover threatened by pasture management. Forest Ecology and Management 261: 1664-1674.

Houghton, RA. 2005. Aboveground biomass and the global carbon balance. Global Change Biology 11:945-958

Jenkins, JC; Chojnacky, DC; Heath, LS; Birdsey, RA. 2003. National-scale biomass estimators for United States trees species. Forest Science 49:12-35.

Ketterings, QM; Noordwijk, CMY; Ambagau, R; Palm, CA. 2001. Reducing uncertainty in the use of allometric biomass equations for predicting aboveground tree biomass in mixed secondary forests. Forest Ecology and Management 146:199-209.

Kie, JG; White, M. 1985. Population dynamics of white-tailed deer (Odocoileusvirginanus) on the Welder wildlife refuge, Texas. Southwestern Naturalist 30:105-118.

McKendry, P. 2002. Energy production from biomass (part 1): overview of biomass. Bioresource Technology 83:37-46.

Martínez-Yrizar, A; Mas, M; Pérez-Jiménez, LA; Sarukhán, J. 1992. Net primary productivity of a tropical deciduous forest ecosystem in western Mexico. Journal of Tropical Ecology 12:169-175.
Mokany, K; Raison, RJ; Prokushkin, SA. 2006. Critical analysis of root:shootratos in terrestrial biomes. Global Change Biology 11:1-13.

Návar, J. 2009a. Allometric equations for tree species and carbon stocks for forests of northwestern Mexico. Forest Ecology and Management 257:427-434.

Návar, J. 2009b. Biomass component equations for Latin American species and groups of species. Annals of Forest Science 66:208-216.

Návar, J. 2010. Measurement and assessment methods of forest aboveground biomass: A literature review and the challenges ahead. In Momba, M; Bux, F. eds. Biomass. Sciyo Open Access to Knowledge. Intechopen. p. 27-64.

Návar-Cháidez, JJ. 2009. Allometric equations and expansion factors for tropical dry trees of eastern Sinaloa, Mexico. Tropical and Subtropical Agroecosystems 10:45-52.

Parolin, P. 2002. Radial gradients in wood specific gravity in trees of Central Amazonian floodplains. IAWA Journal 23:449-457.

Pilli, R; Anfodillo, T; Carrer, M. 2006. Towards a functional and simplified allometry for estimating forest biomass. Forest Ecology and Management 237:583-593.

Schumacher, FX; Hall, FS. 1933. Logarthmic expression of timber-tree volume. Journal of Agricultural Research 47:719-734.

Silva-Arredondo, M; Návar J. 2009. Estimación de factores de expansión de carbono en comunidades templadas del norte de México. Revista Chapingo: Serie Ciencias Forestales y del Ambiente 15(2):155-169.

Ter-Mikaelian, MT; Korzukhin, MD. 1997. Biomass equations for sixty-five North American tree species. Forest Ecology and Management 97:1-24.

West, GB; Brown, JH Enquist, BJ. 1999a. The fourth dimension of live: fractal geometry and allometric scaling of organisms. Science 284:167-169.

West, GB; Brown, JH; Enquist, BJ. 1999b. A general model for the structure and allometry of plant vascular systems. Nature 400:664-667.

Youkhana, AH; Idol, TW. 2011. Allometric models for predicting above- and belowground biomass of Leucaena-KX2 in a shaded coffee agroecosystem in Hawaii. Agroforestry Systems 83:331-345. DOI 10.1007/s10457-011-9403-6.

Zianis, D; Mencuccini, M. 2004. On simplifying allometric analyses of forest biomass. Forest Ecology and Management 187:311-332. 